\title{
A Design Method of the Transmitting Waveform of Electromagnetic Sounding Based on Selective Harmonic Elimination
}

\author{
Lihui Gao ${ }^{(D)},{ }^{1}$ Yongkang Liu ${ }^{D},{ }^{1}$ Nan Chen ${ }^{D},{ }^{1}$ Haolin Li $\left(\mathbb{D},{ }^{2}\right.$ Niaona Zhang ${ }^{D},{ }^{1}$ \\ and Shengbao Yu $\mathbb{D}^{3}$ \\ ${ }^{1}$ College of Electrical and Electronic Engineering, Changchun University of Technology, Changchun 130012, China \\ ${ }^{2}$ State Grid Baishan Power Supply Company, Baishan 134300, China \\ ${ }^{3}$ College of Instrumentation and Electrical Engineering, Jilin University, Changchun 130061, China
}

Correspondence should be addressed to Nan Chen; chennan@ccut.edu.cn

Received 6 August 2021; Revised 4 December 2021; Accepted 20 December 2021; Published 10 January 2022

Academic Editor: Zhiwen Chen

Copyright (c) 2022 Lihui Gao et al. This is an open access article distributed under the Creative Commons Attribution License, which permits unrestricted use, distribution, and reproduction in any medium, provided the original work is properly cited.

\begin{abstract}
The exploration target at different depths through the ground-airborne frequency domain electromagnetic method (GAFDEM) is detected by transmitting waveforms at different frequencies. When taking these different depths into detail, arbitrarily distributed frequencies are needed. However, the current transmitting waveforms are mostly in a fixed frequency ratio or frequency difference, which fails to meet the requirements of exploration accuracy and efficiency at the same time. Therefore, as a solution to this problem, this paper proposes a transmitting waveform design method based on selective harmonic eliminated pulse width modulation (SHEPWM) technology. In the SHEPWM method, three transmitting waveforms with the desired spectrum are obtained by directly controlling the switching angles of a binary sequence with an artificial neural network algorithm. Firstly, our study puts forward the basic theories and principles of the full-periodic asymmetric SHEPWM waveform. Secondly, the study, through simulation, realizes the pseudorandom, depth-focused, and layer-identification waveform with different detection depths. Finally, the application of the proposed SHEPWM waveform to the geological survey in Kaili City, Guizhou Province, proves the correctness and feasibility of this proposed method.
\end{abstract}

\section{Introduction}

The ground-airborne frequency domain electromagnetic method (GAFDEM), a mixture of ground and airborne electromagnetic methods, has been widely used for mineral exploration, geothermal investigation, hydrocarbon exploration, and groundwater survey [1]. A typical GAFDEM system is composed of the ground launch system, air receiving system, and ground remote monitoring system, as shown in Figure 1. The ground launch system radiates electromagnetic fields into space by emitting a single frequency square waveform or a multifrequency pseudorandom square waveform. When the electrical structure of the abnormal structure underground changes, the amplitude and phase of electromagnetic fields will change accordingly. The air receiving system synchronously collects the vertical magnetic field component and records the position, height, and attitude information of the coil at the same time. By detecting the abnormal distribution of space electromagnetic field, the GAFDEM inversely interprets the electric property structure of underground medium $[2,3]$. This method combines the high-power transmission of the ground electromagnetic method and the rapid noncontact acquisition method of the airborne electromagnetic method, which can be used for high efficiency and large depth geological survey in complex terrain conditions.

According to the theory of frequency sounding, changing the frequency of the emission source could alter the distribution of the electromagnetic field in space which can determine the information of the abnormal individual about the position, depth, size, etc. [4]. At present, the GAFDEM detection system mostly uses the $2 n$ 


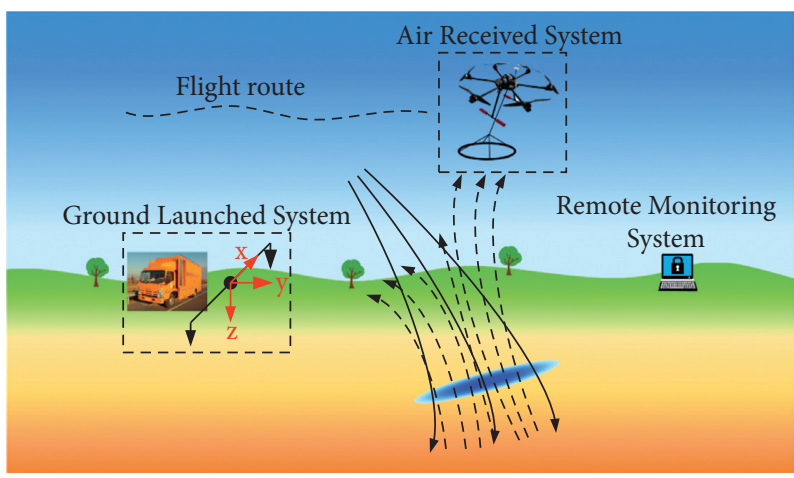

Figure 1: A typical GAFDEM system.

pseudorandom waveform as its transmitting waveform because the $2 n$ pseudorandom waveform could emit multiple frequencies with the same amplitude at a time. However, for the high precision exploration of the targets at specific depths, this method requires repetitive measurements and adjustments of the fundamental frequency to complete the investigative work of all the main frequencies due to the fixed frequency ratio of the $2 n$ pseudorandom waveform. This scheme observes the response of different frequency currents at different times, resulting in different random interference and different signal-to-noise ratios at various frequencies. At the same time, multiple emissions and repeated flight measurements will severely reduce detection efficiency and increase detection costs. Thus, it is important to design a customizable waveform with arbitrarily distributed harmonics for the GAFDEM to improve survey efficiency when detecting different depths or targets.

In order to design the optimal transmitting waveform, researchers have proposed a method to modify the square wave frequency spectrum. Specialized waveforms have been presented by Constable and Cox, patented by ExxonMobil, and researched by Myer et al. [5]. Although all of these waveforms have higher energy at the desired frequencies, they are unsuitable for the high-precision detection of targets at any depth since their harmonic frequencies are not arbitrarily adjustable. Mittet and Schaug proposed a method for designing transmitting waveforms by which current amplitudes as a function of frequency would result in an approximate predefined or desired distribution [6]. This method is based on matching the desired frequency spectra with the spectra obtained from the generalized square waves. The calculating time and cost will rise with the total number of main frequencies.

As a result, this paper proposes a method of transmitting waveform design based on selective harmonic eliminated pulse width modulation (SHEPWM) technology. The steps are as follows: analyzing the principle of the full-period asymmetric SHEPWM, establishing the SHEPWM nonlinear equations to control the amplitude, phase, and DC component of each harmonic according to the frequency domain characteristics of the expected transmitting waveform, and combining the neural network algorithm to obtain the switching instants corresponding to the frequency domain information. This paper uses the corresponding formula to construct the transmitting waveform of controllable frequency domain information which is then applied to electromagnetic sounding to achieve high-precision exploration of targets at specific depths.

\section{The Principle of Full-Periodic Asymmetric SHEPWM}

SHEPWM has been widely applied in the fields of ground power units [7], variable speed drives [8,9], and multilevel converters [10-12] because of its multiple advantages of low switching frequency, more controlled low-order harmonic of the output waveform, and capability of avoiding tripled harmonics. Previous works have made fundamental assumptions that enforce output waveform quarter-wave symmetry, and all the harmonics and DC components are set to zero [13]. However, the harmonic components of the output voltage waveform are useful signals for geophysical electromagnetic surveys. High-energy harmonic components are able to improve the signal-to-noise ratio and imaging accuracy as well as reduce the cost. Therefore, in our study, the full-periodic asymmetric SHEPWM approach is proposed to enhance useful harmonic components and eliminate unnecessary harmonic components.

Figure 2 is the schematic diagram of the full-periodic asymmetrical SHEPWM waveform. $U_{d}$ is the amplitude; $\alpha_{1}$, $\alpha_{2}, \ldots, \alpha_{N}$ are the switching instants, and $N$ is the total number of switching instants in one period, also known as degrees of freedom, which can be an odd or even number. When $N$ is an odd number, the waveform has different polarities at the terminal of the first cycle and the beginning point of the next cycle. In order to ensure the continuity of waveforms with different cycles, $N$ in this article is set to an even number. According to the basic properties of the Fourier series, the Fourier coefficients of the SHEPWM periodic waveform are 


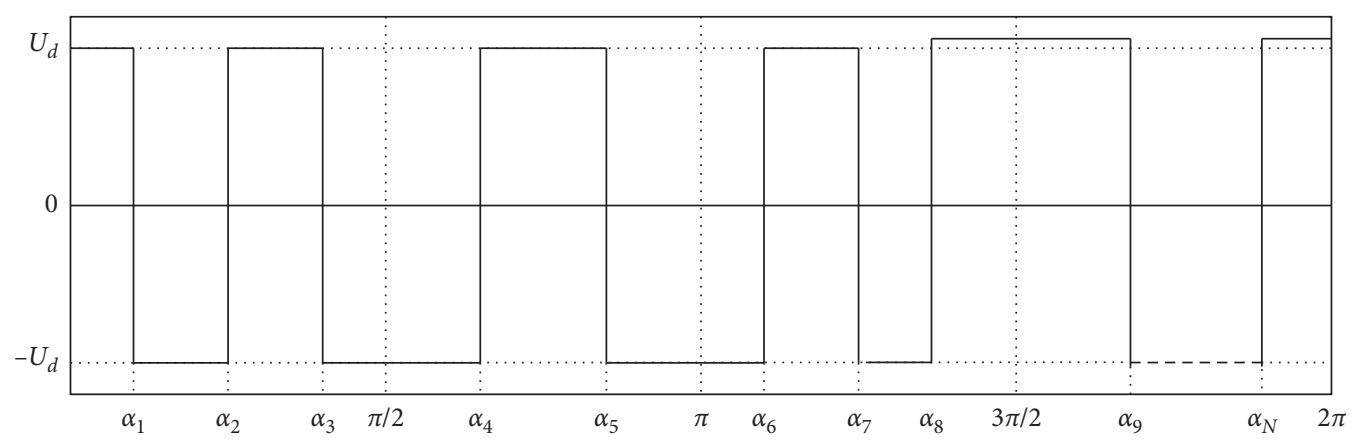

FIGURE 2: Schematic diagram of the full-periodic asymmetric SHEPWM waveform.

$$
\left\{\begin{array}{l}
a_{S 0}=\frac{2 U_{d}}{\pi}\left[\pi+\sum_{k=1}^{N}(-1)^{k+1} \alpha_{k}\right], \\
a_{S i}=\frac{2 U_{d}}{i \pi} \sum_{k=1}^{N}(-1)^{k} \cos \left(i \alpha_{k}\right) \quad i=1,2,3 \ldots \\
b_{S i}=\frac{2 U d}{i \pi} \sum_{k=1}^{N}(-1)^{k+1} \sin \left(i \alpha_{k}\right),
\end{array}\right.
$$

where $i$ is the number of harmonics.

After the frequency domain information of the expected output waveform is determined, the Fourier coefficients of the SHEPWM periodic waveform are matched with the frequency domain information of the expected output waveform to obtain full-periodic asymmetric SHEPWM nonlinear equations:

$$
\left\{\begin{array}{l}
\frac{2 U_{d}}{\pi}\left[\pi+\sum_{k=1}^{N}(-1)^{k+1} \alpha_{k}\right]=A_{E 0} \\
\frac{2 U_{d}}{i \pi} \sum_{k=1}^{N}(-1)^{k} \cos \left(i \alpha_{k}\right)=A_{E i} \cos \theta_{E i} \quad i=1,2,3 \ldots \\
\frac{2 U_{d}}{i \pi} \sum_{k=1}^{N}(-1)^{k+1} \sin \left(i \alpha_{k}\right)=A_{E i} \sin \theta_{E i}
\end{array}\right.
$$

where $A_{E 0}$ is the DC component of expected output voltage; $A_{E i}$ is the $i$ th harmonic amplitude of expected output voltage; and $\theta_{E i}$ is the $i$ th harmonic phase of expected output voltage.

The left side of equation (2) is the polynomial related to the switching instants $\alpha_{1}, \alpha_{2}, \ldots, \alpha_{N}$, and the right side is the polynomial related to the controlled DC component $A_{E 0}$, harmonic amplitude $A_{E i}$, and phase $\theta_{E i}$ of the output voltage. Therefore, by solving equation (2), the switching instants that meet the emission requirements can be accurately calculated.

Equation (2) has $N$ degrees of freedom if the switching instants are independent of each other. In calculation, each harmonic component requires two degrees of freedom
$(N=2)$ to control because it contains amplitude and phase information. The DC component requires one degree of freedom $(N=1)$ to control. The relationship between the harmonic order $i$ and the degree of freedom $N$ is

$$
N=2(i+1) \text {. }
$$

Combining equations (2) and (3) can be simplified as

$$
\left\{\begin{array}{l}
\sum_{k=1}^{2(i+1)}(-1)^{k+1} \alpha_{k}=\frac{\pi}{2 U_{d}}\left(A_{E 0}-2 U_{d}\right) \\
\sum_{k=1}^{2(i+1)}(-1)^{k} \cos \left(i \alpha_{k}\right)=\frac{\pi}{2 U_{d}} i A_{E i} \cos \theta_{E i} \quad i=1,2,3 \ldots \\
\sum_{k=1}^{2(i+1)}(-1)^{k+1} \sin \left(i \alpha_{k}\right)=\frac{\pi}{2 U_{d}} i A_{E i} \sin \theta_{E i}
\end{array}\right.
$$

In addition, the switching instants calculated using equation (4) must satisfy the following relationship:

$$
0<\alpha_{1}<\alpha_{2}<\cdots<\alpha_{N}<2 \pi
$$

\section{Transmitting Waveform Design Method Based on SHEPWM for GAFDEM}

3.1. Pseudorandom Waveform Obtained by SHEPWM. The $2 n$ pseudorandom waveform is able to distribute its energy relatively evenly to several main frequencies. There are $n$ main frequencies in the $2 n$ pseudorandom waveform with harmonic orders $2^{0}, 2^{1}, 2^{2}, \ldots, 2^{n-1}$, respectively. The main frequencies are arranged in $2 n$, which means that they are arranged equidistantly in logarithmic coordinates with a fixed ratio of 2 . These main frequencies have almost equivalent amplitudes and a larger sum accounting for the most of signal total energy. Thus, the $2 n$ pseudorandom wave emitted in one detection flight contains multiple main frequencies, which contributes to a higher detection efficiency. In recent years, the advantageous $2 n$ pseudorandom waveform has become one of the standard transmitting waveforms in the transmission system for the frequency domain electromagnetic methods $[14,15]$. 
In order to verify the correctness and effectiveness of the SHEPWM method, a pseudorandom five-frequency waveform based on the SHEPWM method is presented and compared with the standard pseudorandom five-frequency waveform. Since the main frequencies of the standard pseudorandom five-frequency waveform are the $1^{\text {st }}, 2^{\text {nd }}, 4^{\text {th }}$, $8^{\text {th }}$, and $16^{\text {th }}$ harmonics, the corresponding SHEPWM nonlinear equations should have 34 switching instants, that is, $N=34$. The settings of the frequency domain information for the SHEPWM pseudorandom five-frequency waveform are shown in Table 1 . The DC component is set to zero. The inverter DC side voltage value $U_{d}$ is set to $1 \mathrm{~V}$. Substituting the above frequency domain information into equation (4), the SHEPWM pseudorandom five-frequency waveform nonlinear equations can be obtained as

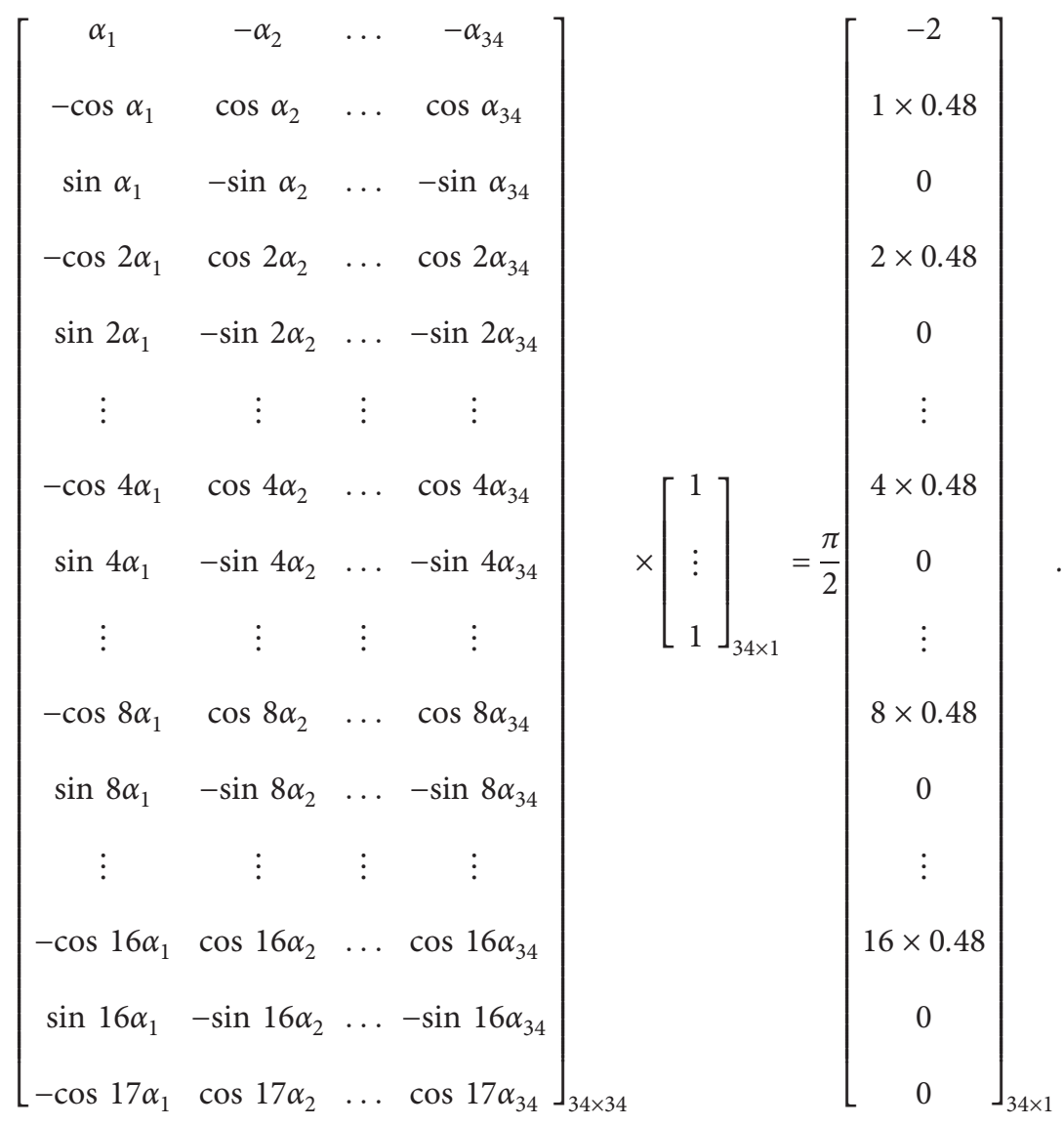

It is worth noting that there are two principles to be followed when using the SHEPWM method to design the transmitting waveform. Firstly, the maximum $A_{s i}$ should not be greater than $4 U_{d} / \pi$ because this value is the fundamental amplitude of the bipolar square waveform. Secondly, all $A_{s \mathrm{i}}$ should conform to the energy conservation law as follows:

$$
\sum_{i=1}^{+\infty}\left(\frac{A_{s i}}{\sqrt{2}}\right)^{2}=\left(U_{d}\right)^{2} .
$$

Usually, the SHEPWM nonlinear equations are solved by two approaches. On the one hand, the first approach proposes numerical iterative techniques such as Newton-Raphson and Gauss-Newton [16, 17]. But these methods require the selection of the initial point to be very close to the exact solution or will lead to the solution unconverged. On the other hand, the second approach overcomes these drawbacks and finds the global optimum solution within a short time searching $[18,19]$. This approach employs analytical equations to form an objective function, which is then minimized to achieve the solutions. In our study, we use the second approach and solve the equations by using an artificial neural network (ANN) algorithm [20]. According to the research conclusion of the literature [20], the switching instants of equation (6) are shown in Table 2.

According to Table 2, the simulation results of the time domain waveform and frequency domain information of the SHEPWM pseudorandom five-frequency waveform are given, as shown in Figure 3. By observing Figure 3(a), the fundamental frequency of the SHEPWM pseudorandom five-frequency waveform is $16 \mathrm{~Hz}$, the amplitude is $1 \mathrm{~V}$, and there are 34 discontinuities in the cycle. By observing Figure $3(\mathrm{~b})$, the amplitudes of the $1^{\text {st }}, 2^{\text {nd }}, 4^{\text {th }}, 8^{\text {th }}$, and $16^{\text {th }}$ harmonics of the SHEPWM pseudorandom five-frequency waveform are larger and basically equal, while the 
TABLE 1: The settings of the frequency domain information for SHEPWM pseudorandom five-frequency waveform.

\begin{tabular}{lc}
\hline $\begin{array}{l}\text { Harmonic } \\
\text { order }\end{array}$ & $1 \sim 16$ \\
\hline Magnitude $(A)$ & $(0.48,0.48,0,0.48,0,0,0,0.48,0,0,0,0,0,0,0$, \\
& $0.48)$ \\
Phase $\left(^{\circ}\right)$ & $(0,0,0,0,0,0,0,0,0,0,0,0,0,0,0,0)$ \\
\hline
\end{tabular}

amplitudes of other subharmonics except the main frequency are smaller. The simulation results are consistent with the preset values and standard pseudorandom fivefrequency waveform, which verifies the correctness of the design method for the SHEPWM pseudorandom waveform.

3.2. Depth-Focused Waveform Obtained by SHEPWM. In recent years, the growing need for exploration accuracy prompts the demand for high-precision exploration for specific depth targets. However, for pseudorandom waveforms, the frequency ratio of adjacent main frequencies is fixed, which limits its exploration effect on targets at specific depths. Therefore, this paper presents the design method of the SHEPWM depth-focused waveform, improving the exploration accuracy of targets at specific depths by transmitting waveforms with a concentrated distribution of main frequencies. The main frequency of the SHEPWM depthfocused waveform is set as the 7 th, $8^{\text {th }}$, and 9 th harmonics, and so the corresponding SHEPWM nonlinear equations should have 20 switching instants, that is $N=20$. The settings of the frequency domain information for the SHEPWM depth-focused waveform are shown in Table 3. The DC component is set to zero. The inverter DC side voltage value $U_{d}$ is set to $1 \mathrm{~V}$. Substituting the above frequency domain information into equation (4), the SHEPWM depth-focused waveform nonlinear equations can be obtained as

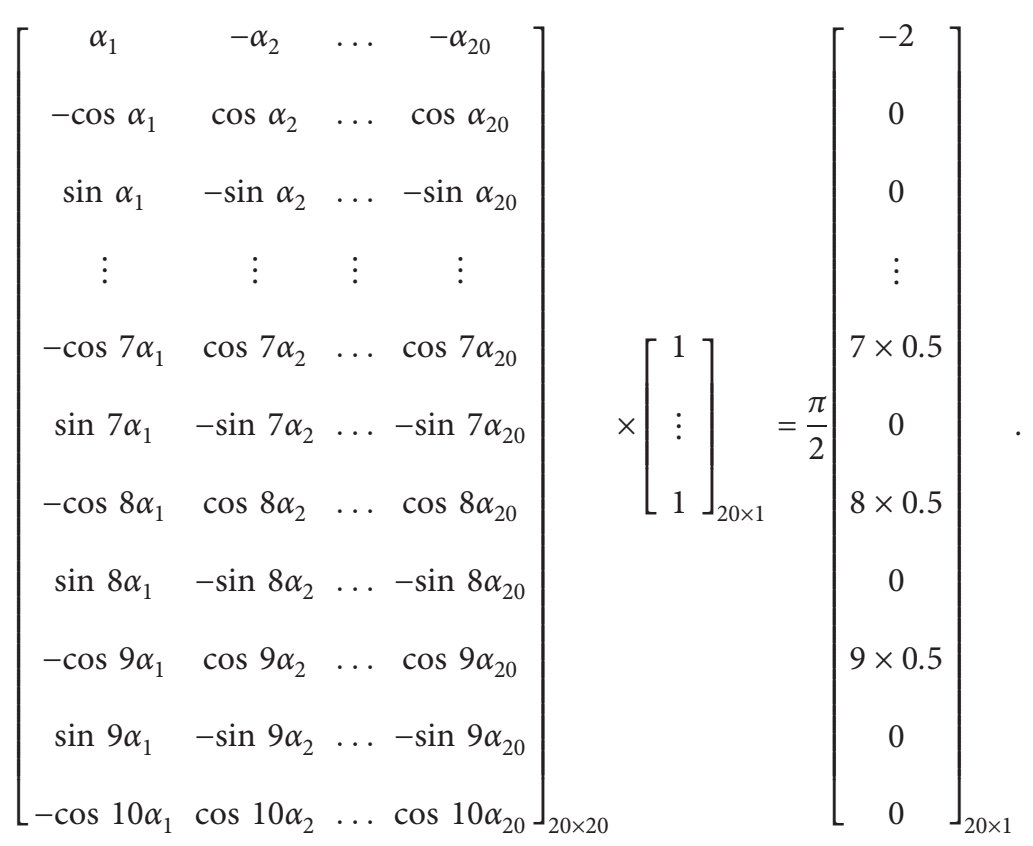

Furthermore, the fundamental frequency of the SHEPWM waveform can be set in combination with geological data and the skin depth formula (9) to improve the exploration accuracy of targets at specific depths.

$$
\delta=-503 \sqrt{\frac{\rho}{f}}
$$

where $\rho$ is the average apparent resistivity in the survey area, and $f$ is the survey frequency.

Table 4 shows the solution of equation (8). Figure 4 shows the simulation results of the SHEPWM depth-focused waveform. By observing Figure 4(a), the fundamental frequency of the SHEPWM depth-focused waveform is $16 \mathrm{~Hz}$, the amplitude is $1 \mathrm{~V}$, and there are 20 discontinuities in the cycle. By observing Figure 4(b), the amplitudes of the 7th, 8th, 9th, 10th, and 11th harmonics of the SHEPWM depth- focused waveform are larger, while the amplitudes of other harmonics are smaller. It is worth noting that the 10th and 11th harmonics are uncontrollable harmonics, which can be included in the main frequency range due to their large amplitude. The simulation results are consistent with the preset values, which verify the correctness of the design method for the SHEPWM depth-focused waveform.

\subsection{Layer-Identification Waveform Obtained by SHEPWM.} The Earth's interior is layered. According to the principle of electromagnetic sounding, the main frequency interval distribution of the transmitting current is required for continuous formation exploration. At the same time, electromagnetic waveforms will cause energy loss when propagating in the formation, and the attenuation depends on the 
TABLE 2: Switching instants for equation (6).

\begin{tabular}{llcll}
\hline \multicolumn{4}{c}{ Switching instants $\alpha_{1} \sim \alpha_{34}(\mathrm{rad})$} \\
\hline 0.3066 & 0.3217 & 0.6286 & 0.6922 & 1.1173 \\
1.2113 & 1.3584 & 1.5145 & 1.9294 & 1.9867 \\
2.1522 & 2.3553 & 2.5033 & 2.6787 & 3.7426 \\
3.0390 & 3.0837 & 3.1773 & 3.5097 & 3.5732 \\
3.7673 & 3.9165 & 4.1287 & 4.2858 & \\
4.7664 & 4.9225 & 5.0694 & 5.1625 & 5.3494 \\
5.6523 & 5.9583 & 5.9737 & 6.2809 & \\
\hline
\end{tabular}

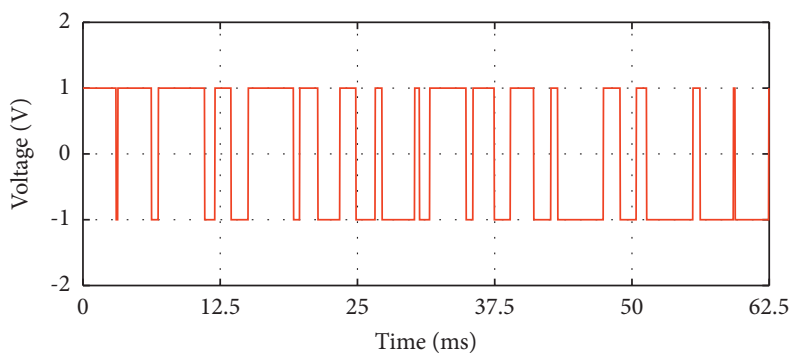

(a)

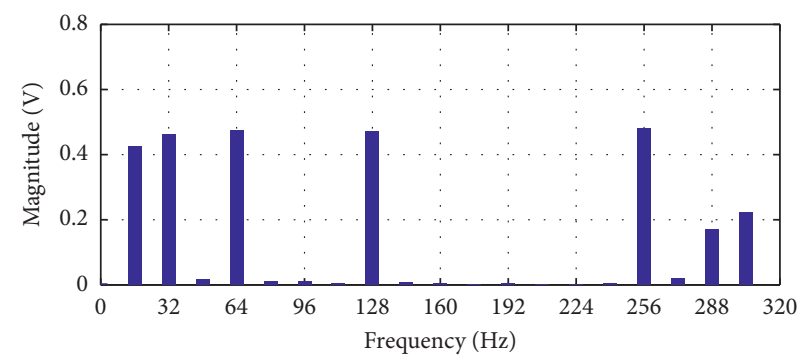

(b)

FIGURE 3: Simulation results of the SHEPWM pseudorandom five-frequency waveform (fundamental frequency is $16 \mathrm{~Hz}$ ). (a) Time domain waveform; (b) frequency domain information.

conductivity of the formation, so the dominant frequency energy of the transmitting current should be determined according to the geological structure of the exploration target. Therefore, for the exploration targets that change layer by layer, this paper presents the design method of the SHEPWM layer-identification waveform. The main frequency of the SHEPWM layer-identification waveform is set as the 1 st, 8 th, $16^{\text {th }}$, and 25 th harmonics, so the corresponding SHEPWM nonlinear equations should have 52 switching instants, that is $N=52$. The settings of the frequency domain information for the SHEPWM layeridentification waveform are shown in Table 5. The DC component is set to zero. The inverter DC side voltage value $U_{d}$ is set to $1 \mathrm{~V}$. Substituting the above frequency domain information into equation (4), the SHEPWM layer-identification waveform nonlinear equations can be obtained as

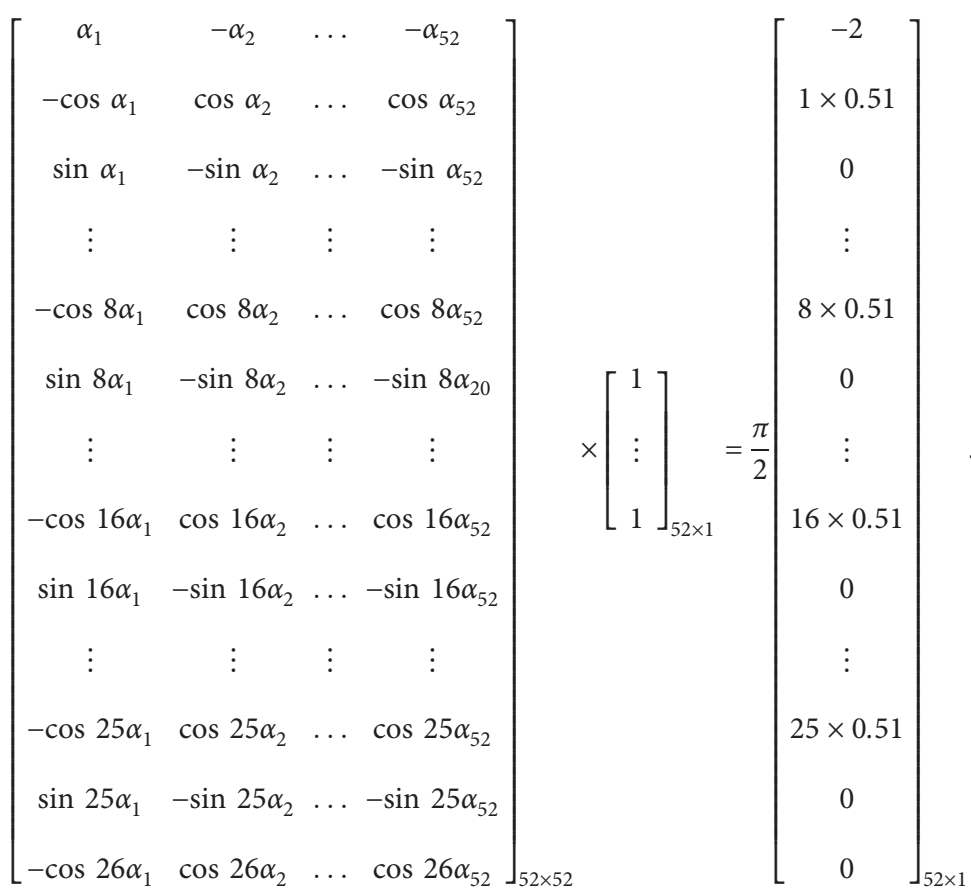


TABle 3: The settings of the frequency domain information for SHEPWM depth-focused waveform.

\begin{tabular}{lc}
\hline Harmonic order & $1 \sim 9$ \\
\hline Magnitude $(A)$ & $(0,0,0,0,0,0,0.5,0.5,0.5)$ \\
Phase $\left({ }^{\circ}\right)$ & $(0,0,0,0,0,0,0,0,0)$ \\
\hline
\end{tabular}

Similarly, the fundamental frequency of the SHEPWM waveform can be set in combination with geological data and the skin depth equation (9) to improve the exploration accuracy of targets at specific depths.

Table 6 shows the solution of equation (10). Figure 5 shows the simulation results of the SHEPWM layer-identification waveform. By observing Figure 5(a), the fundamental frequency of the SHEPWM layer-identification waveform is $16 \mathrm{~Hz}$, the amplitude is $1 \mathrm{~V}$, and there are 52 discontinuities in the cycle. By observing Figure 5(b), the amplitude value of the 1st, 8 th, 16th, and 25th harmonics of the SHEPWM layer-identification waveform is $0.51 \mathrm{~V}$. The simulation results are consistent with the preset values, which verify the correctness of the design method for the SHEPWM layer-identification waveform.

3.4. Waveform Comparison. Figure 6 is a comparison of the frequency coverage of the three waveforms discussed above. For each waveform, we plot the electromagnetic sounding coverage corresponding to the dominant frequency. The fundamental frequency of each waveform is the same at $16 \mathrm{~Hz}$. The average apparent resistivity was selected to be $10 \Omega \mathrm{m}$.

For the SHEPWM pseudorandom waveform, the main frequencies were $\{16,32,64,128,256\} \mathrm{Hz}$ and the corresponding apparent depths were $\{-398,-281,-199,-141$, -99\} $m$. The exploration depth of the pseudorandom waveform is from $-99 \mathrm{~m}$ to $-398 \mathrm{~m}$, and the depth distribution is relatively uniform. For the SHEPWM layeridentification waveform, the main frequencies were $\{16,128$, $256,400\} \mathrm{Hz}$ and the corresponding apparent depths were $\{-398,-141,-99,-80\} \mathrm{m}$. The exploration depth of layeridentification waveform is from $-80 \mathrm{~m}$ to $-398 \mathrm{~m}$. Compared with the pseudorandom waveform, the exploration depth range is expanded by $20 \mathrm{~m}$, but there are exploration blind areas in the deep. For the SHEPWM depth-focused waveform, the main frequencies were $\{112,128,144,160,176\} \mathrm{Hz}$ and the corresponding apparent depths were $\{-150,-141$, $-133,-126,-120\} m$. The exploration depth of the depthfocused waveform is from $-120 \mathrm{~m}$ to $-150 \mathrm{~m}$. Compared with the pseudorandom and layer-identification waveforms, the exploration depth range is greatly reduced, but there are five effective dominant frequencies in this depth range, which can realize accurate exploration in this depth range.

According to the above analysis, when conducting a geological survey, the pseudorandom waveform and the layer-identification waveform can be firstly used to realize a wide range of depth surveys. Then, according to the exploration results, the appropriate fundamental frequency depth-focused waveform is selected to accurately explore the depth of interest.

\section{Field Test of SHEPWM Waveform for GAFDEM}

4.1. Survey Area and Transmitting Process. In order to verify the feasibility of the design method of the SHEPWM waveform, a GAFDEM field test was conducted on WanliQinggangao Coal Mine in Kaili City, Guizhou Province. Wanli-Qinggangao coal mine is located in the Pinglu river basin. There is a large amount of water in the goaves of the two coal mines, and the acidic wastewater is discharged to the Pinglu river in the form of springs along the underground karst pipes, which seriously pollutes the river water, as shown in Figure 7. The Wanli-Qinggangao coal mine is in a state of shutdown due to the need of environmental management of the Pinglu river.

Figure 8 shows the general view of the test site. The coordinates of the emission source electrode are $26.665682^{\circ}$, $107.831063^{\circ}$ and $26.658387^{\circ}, 107.819157^{\circ}$. The distance between the emission source and the survey area is approximately $2.3 \mathrm{~km}$. There are 52 survey lines in the survey area, each of which has a length of about $1.03 \mathrm{~km}$, the survey line spacing is approximately $20 \mathrm{~m}$, and the survey area length is approximately $1.1 \mathrm{~km}$. The total area of the survey area is approximately $1 \mathrm{~km}^{2}$. To simplify the description, we predefined that the forward direction is from point a to point $b$ and that the return direction is from point $b$ to point a in each measurement flight.

In the field test, we investigated the underground structure along the survey line by transmitting the SHEPWM waveform. Figure 9 shows the three types of SHEPWM waveforms. The fundamental frequency of Figures 9(a) and 9 (d) was $320 \mathrm{~Hz}$, the main frequency was the 7th, 8th, 9th, 10th, and 11th harmonics, and the transmitting frequencies were $\{2240,2560,2880,3200,3520\}$ $\mathrm{Hz}$. The fundamental frequency of Figures 9(b) and 9(e) was $512 \mathrm{~Hz}$, the main frequency was the 5th, 7th, 9th, and 11th harmonics, and the transmitting frequencies were $\{2560$, $3584,4608,5632\} \mathrm{Hz}$. The fundamental frequency of Figures 9(c) and 9(f) was $512 \mathrm{~Hz}$, the main frequency was the 7 th, 8th, 9th, 10th, and 11th harmonics, and the transmitting frequencies were $\{3584,4096,4608,5120,5632\} \mathrm{Hz}$.

The acquisition system for the field test was suspended under a six-rotor unmanned aerial vehicle (UAV) at a distance of $15 \mathrm{~m}$. The UAV flew at a height of $50 \mathrm{~m}$ at $5 \mathrm{~m} / \mathrm{s}$. During the transmitting process, we collected the vertical magnetic field in the air at a sampling rate of $32 \mathrm{kHz}$. After data acquisition, firstly, we used the fast Fourier transform method to obtain the frequency spectrum of the signal. Secondly, we calculated the normalized magnetic field amplitude by dividing the spectral data by the electric moment of the emission source and the sensitivity coefficient of the coil sensor. Finally, we calculated the apparent resistivity and apparent depth at different frequencies to obtain the apparent resistivity diagram. These works were similar to the work reported in our previous article [21].

4.2. Field Test Results. Finally, three-dimensional processing is performed on the detection data to obtain a $3 \mathrm{D}$ apparent 
TABLE 4: Switching instants for equation (8).

\begin{tabular}{llcll}
\hline & \multicolumn{3}{c}{ Switching instants $(\mathrm{rad})$} & \\
\hline 0.3941 & 0.7848 & 1.1265 & 1.3596 & \\
1.7926 & 2.1053 & 2.4063 & 2.6602 & \\
3.2352 & 3.5793 & 3.9082 & 4.1911 & \\
4.7282 & 5.0862 & 5.4772 & 5.8726 & 4.4456 \\
\hline
\end{tabular}

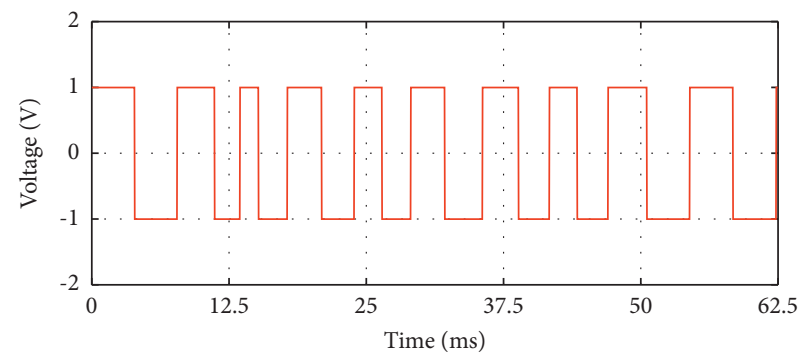

(a)

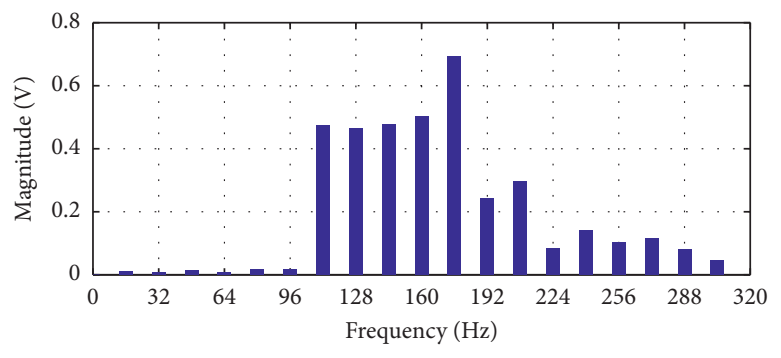

(b)

Figure 4: Simulation results of SHEPWM depth-focused waveform (fundamental frequency is $16 \mathrm{~Hz}$ ). (a) Time domain waveform; (b) frequency domain information.

TABLE 5: The settings of the frequency domain information for SHEPWM layer-identification waveform.

\begin{tabular}{lc}
\hline Harmonic order & $1 \sim 25$ \\
\hline Magnitude $(A)$ & $(0.51,0,0,0,0,0,0,0.51,0,0,0,0,0,0,0,0.51,0,0,0,0,0,0,0,0,0.51)$ \\
Phase $\left(^{\circ}\right)$ & $(0,0,0,0,0,0,0,0,0,0,0,0,0,0,0,0,0,0,0,0,0,0,0,0,0)$ \\
\hline
\end{tabular}

TABle 6: Switching instants for equation (10).

\begin{tabular}{|c|c|c|c|c|}
\hline \multicolumn{5}{|c|}{ Switching instants (rad) } \\
\hline 0.1883 & 0.2425 & 0.3588 & 0.4442 & 0.5654 \\
\hline 0.6717 & 0.6984 & 0.7589 & 1.0685 & 1.1112 \\
\hline 1.2207 & 1.2664 & 1.3883 & 1.5121 & 1.8979 \\
\hline 1.9592 & 2.1178 & 2.2041 & 2.2529 & 2.3339 \\
\hline 2.5831 & 2.6138 & 2.7047 & 2.7862 & 2.9043 \\
\hline 3.0939 & 3.1796 & 3.2365 & 3.4011 & 3.5364 \\
\hline 3.6282 & 3.8726 & 3.9006 & 4.0016 & 4.1389 \\
\hline 4.2982 & 4.3550 & 4.4121 & 4.4233 & 4.7742 \\
\hline 4.9015 & 5.0481 & 5.1038 & 5.2173 & 5.2459 \\
\hline 5.5394 & 5.6899 & 5.8025 & 5.8871 & 6.0059 \\
\hline 6.0725 & 6.2804 & & & \\
\hline
\end{tabular}

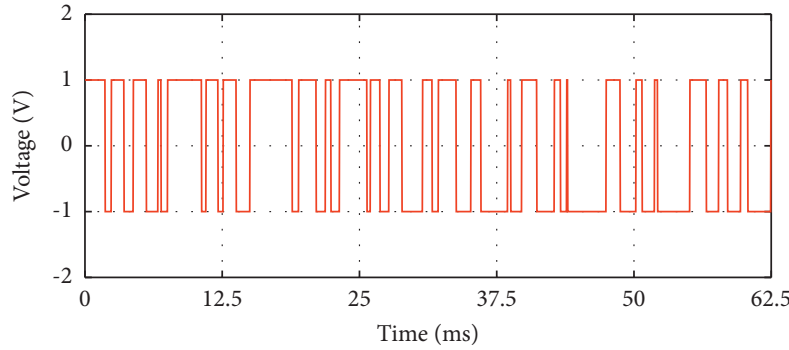

(a)

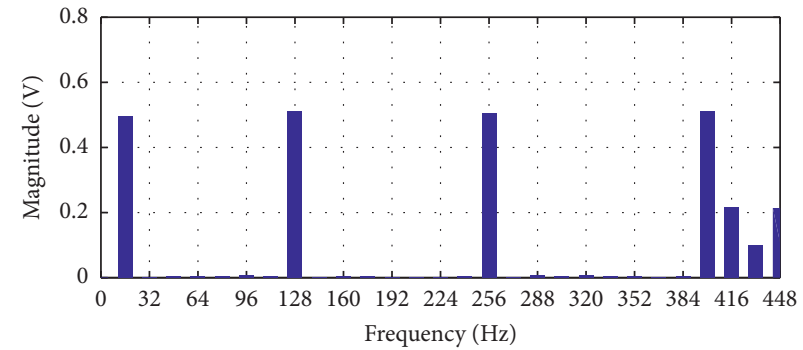

(b)

FIGURE 5: Simulation results of SHEPWM layer-identification waveform (fundamental frequency is $16 \mathrm{~Hz}$ ). (a) Time domain waveform; (b) frequency domain information. 


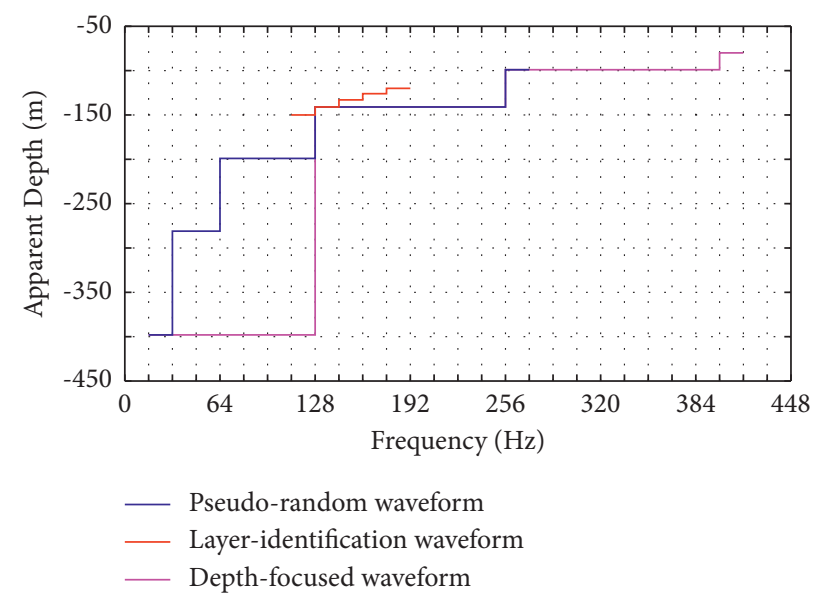

FIgURE 6: Comparison of the frequency coverage of the three waveforms.

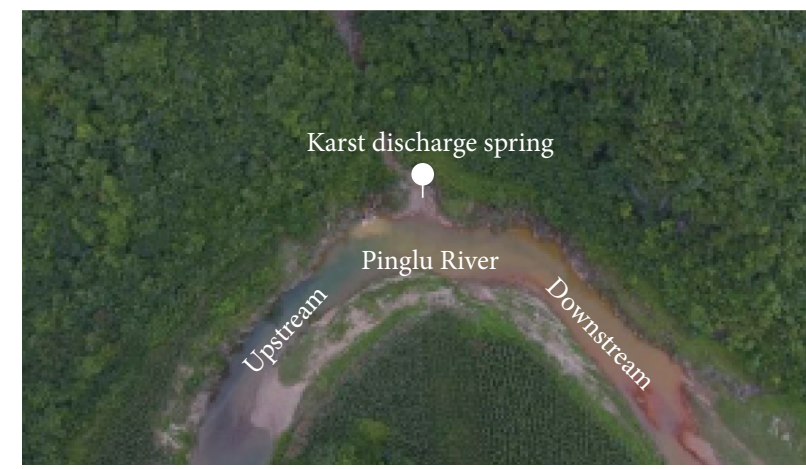

Figure 7: Water pollution in Pinglu river.

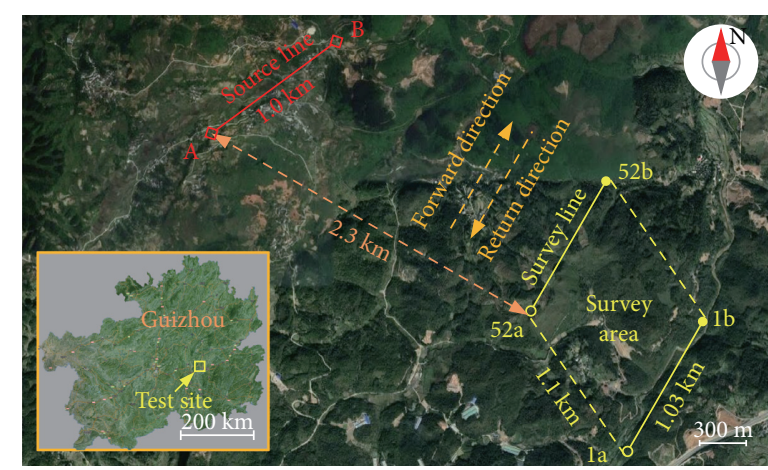

Figure 8: General view of the test site.

resistivity map, as shown in Figure 10(a). In Figure 10(a), the horizontal axis represents the width of measuring area (the length of the measuring line), while the longitudinal axis represents the length of the measuring area, and the vertical axis represents the altitude of the measuring area. Figure 10(b) shows the apparent resistivity map with elevation $480 \mathrm{~m}$ above sea level. It can be observed that this map contains 9 low-resistance anomalies, of which there are 2 low-resistance anomalies with a larger range and 7 lowresistance anomalies with a smaller range. Combined with the original geological data for comprehensive analysis, as shown in Figure 11, the extension direction of the low resistivity anomaly is consistent with that of the karst cave. Therefore, it is judged that the low-resistance anomaly No. 1 is a cave, and the low-resistance anomaly No. 2 is conjectured to be a mined-out area. 


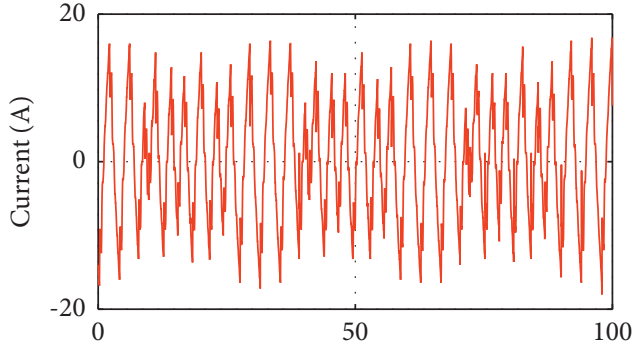

(a)

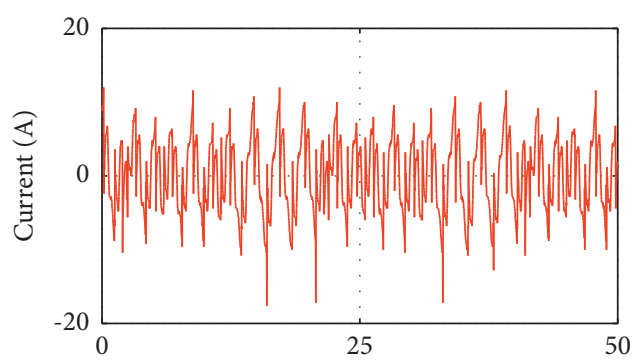

(c)

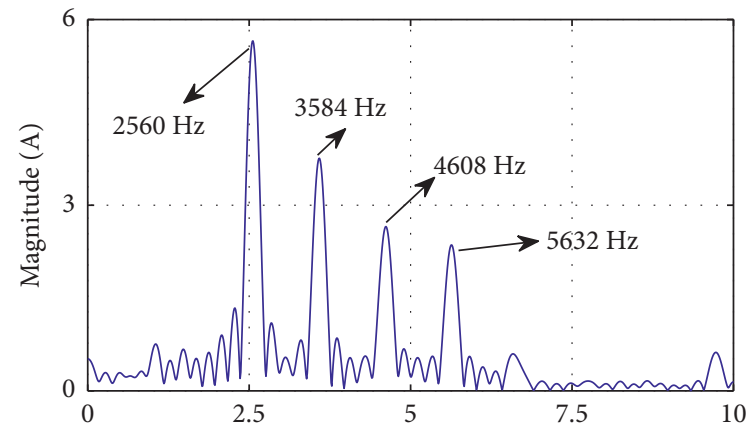

(e)

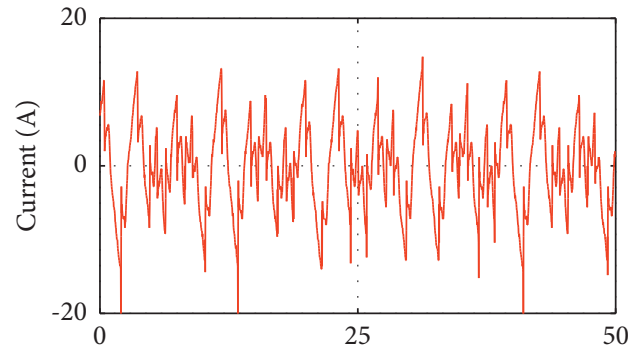

(b)

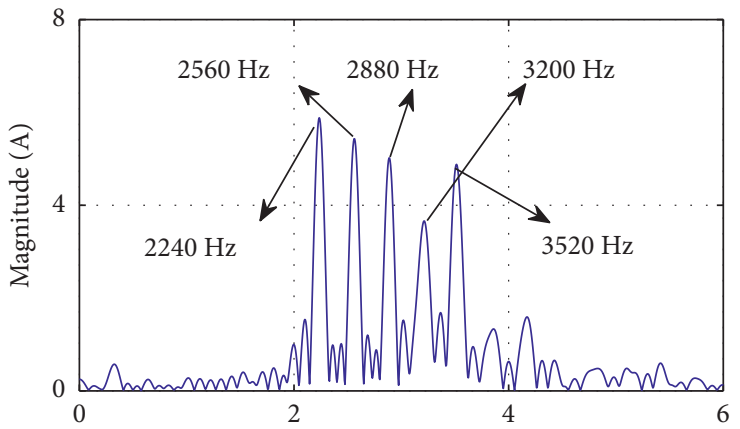

(d)

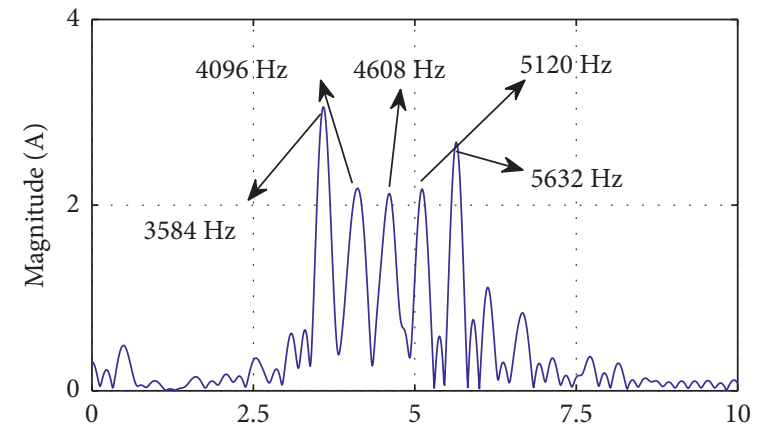

(f)

FIgURE 9: SHEPWM waveforms in the field test. (a-c) Time domain waveform; (d-f) frequency domain information.

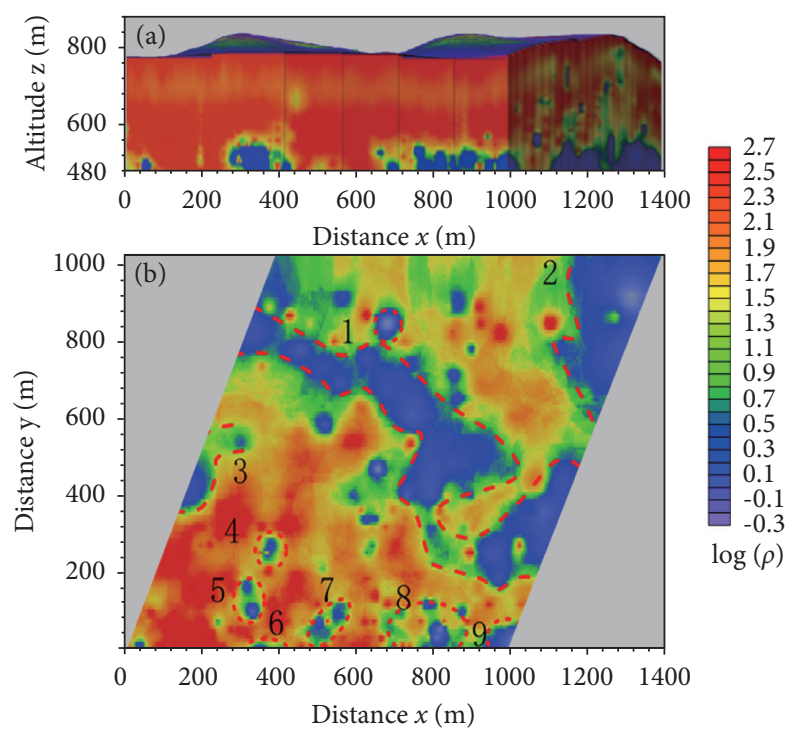

FIGURE 10: Apparent resistivity map. (a) 3D apparent resistivity map; (b) apparent resistivity map with elevation $480 \mathrm{~m}$ above the sea level. 


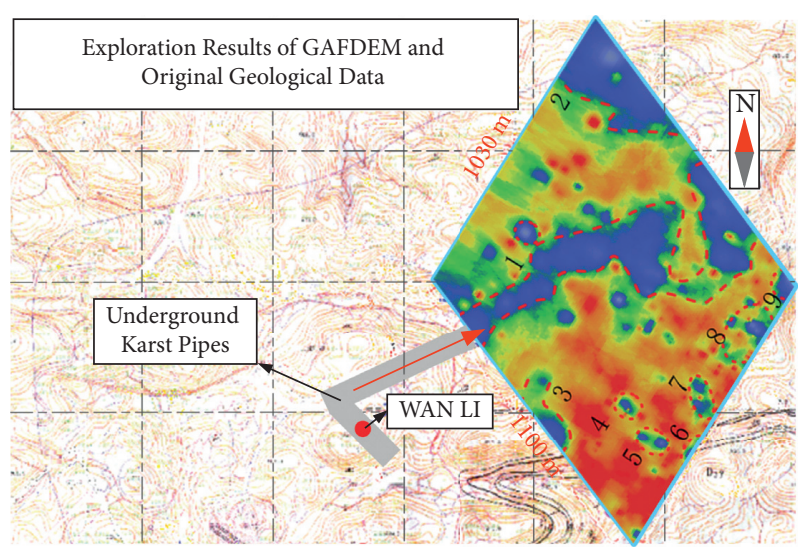

FIGURE 11: Comprehensive resistivity result graph.

\section{Conclusion}

In this paper, we design three transmitting waveforms of which the main frequencies are assembled and concentrated to the different depth ranges based on the principle of frequency sounding, thus improving the exploration efficiency and accuracy of specific depth targets. These waveforms are implemented via the SHEPWM method as follows: setting the Fourier coefficients of the SHEPWM waveform to equal with the frequency domain information of the expected transmitting waveform, combining with the neural network algorithm to control the switching angle of the binary sequence, and finally achieving customization of the energy and distribution of these main frequencies. The simulation and experimental results show that the SHEPWM waveform has higher survey accuracy, which is more conducive to the high-precision electromagnetic survey of specific depth targets. At present, this method has successfully been applied in GAFDEM.

\section{Data Availability}

The Matlab/Simulink model and XLSX data used to support the findings of this study are available from the corresponding author upon request.

\section{Conflicts of Interest}

The authors declare that they have no conflicts of interest.

\section{Acknowledgments}

This work was supported by the "Development of Helicopter Aeroelectromagnetic Measurement Technology System" (the National Key R\&D Program of China, Grant No. 2017YFC0601802).

\section{References}

[1] L. L. Kang, L. C. Liu, C. C. Liu, F. Zhou, and Z. Shi, "Forward modeling and analyzing for frequency domain semi-airborne em method," in Proceedings of the International Workshop and Gravity, Electrical \& Magnetic Methods and their Applications, pp. 366-369, Chenghu, China, April 2015.
[2] T. Mogi, Y. Tanaka, K. Kusunoki et al., "Development of grounded electrical source airborne transient EM (GREATEM)," Exploration Geophysics, vol. 29, no. 2, pp. 61-64, 1998.

[3] R. S. Smith, A. P. Annan, and P. D. Mcgowan, "A comparison of data from airborne, semi-airborne, and ground electromagnetic systems," Geophysics, vol. 66, no. 5, pp. 1379-1385, 2001.

[4] B. R. Spies and F. C. Frischknecht, "5. Electromagnetic sounding," Electromagnetic Methods in Applied Geophysics, vol. 2, pp. 285-425, 1991.

[5] D. Myer, S. Constable, and K. Key, "Broad-band waveforms and robust processing for marine CSEM surveys," Geophysical Journal International, vol. 184, no. 2, pp. 689-698, 2011.

[6] R. Mittet and T. P. Schaug, "Shaping optimal transmitter waveforms for marine CSEM surveys," Geophysics, vol. 73, no. 3, pp. 97-104, 2008.

[7] U. B. Jensen, F. Blaabjerg, and J. K. Pedersen, "A new control method for $400-\mathrm{Hz}$ ground power units for airplanes," IEEE Transactions on Industry Applications, vol. 36, no. 1, pp. $180-187,2000$.

[8] S. R. Bowes, "Advanced regular-sampled PWM control techniques for drives and static power converters," IEEE Transactions on Industrial Electronics, vol. 42, no. 4, pp. 367-373, 1995.

[9] S. R. Bowes and P. R. Clark, "Regular-sampled harmonicelimination PWM control of inverter drives," IEEE Transactions on Power Electronics, vol. 10, no. 5, pp. 521-531, 1995.

[10] B. Diong, K. Corzine, and S. L. Basireddy, "Multilevel inverter-based dual-frequency power supply," IEEE Power Electronics Letters, vol. 1, no. 4, pp. 115-119, 2003.

[11] R. P. Sridhari, K. Georgios, and G. A. Vassilios, "Hybrid seven-level cascaded active neutral - point - clamped - based multilevel converter under SHE-PWM," IEEE Transactions on Industrial Electronics, vol. 60, no. 11, pp. 4794-4804, 2013.

[12] M. H. Etesami, N. Farokhnia, and S. H. Fathi, "Colonial competitive algorithm development toward harmonic minimization in multilevel inverters," IEEE Transactions on Industrial Informatics, vol. 11, no. 2, pp. 459-466, 2015.

[13] J. Cheng, D. Chen, and G. Chen, "Modeling and compensation for dead-time effect in high power IGBT/IGCT converters with SHE-PWM modulation," Energies, vol. 13, no. 17, 2020.

[14] J. S. He, "Closed addition in a three-element set and $2 \mathrm{n}$ sequence pseudorandom signal coding," Journal of Central South University, vol. 41, no. 2, pp. 632-637, 2010.

[15] H. G. Zhou, K. C. Xue, J. Lin et al., "Cascaded transmitter with output of $2 \mathrm{n}$ sequence pseudo random waveform for semiairborne frequency-domain electromagnetic exploration," in Proceedings of the International Workshop and Gravity, Electrical \& Magnetic Methods and their Applications, pp. 19-22, Chenghu, China, April 2015.

[16] H. S. Patel and R. G. Hoft, "Generalized techniques of harmonic elimination and voltage control in thyristor inverters: Part I--Harmonic elimination," IEEE Transactions on Industry Applications, vol. IA-9, no. 3, pp. 310-317, 1973.

[17] H. S. Patel and R. G. Hoft, "Generalized techniques of harmonic elimination and voltage control in thyristor inverters: Part II --- voltage control techniques," IEEE Transactions on Industry Applications, vol. IA-10, no. 5, pp. 666-673, 1974.

[18] S. Marcin, J. Włodzimierz, and S. Adam, "Application of grasshopper optimization algorithm for selective harmonics elimination in low-frequency voltage source inverter," Energies, vol. 13, no. 23, 2020. 
[19] S. S. Lee, B. Chu, N. R. N. Idris, H. H. Goh, and Y. E. Heng, "Switched-battery boost-multilevel inverter with ga optimized SHEPWM for standalone application," IEEE Transactions on Industrial Electronics, vol. 63, no. 4, pp. 2133-2142, 2016.

[20] L. H. Gao, S. B. Yu, C. X. Jiang, N. Chen, R. Chen, and $\mathrm{H}$. Yong, "Application of SHEPWM in helicopter transient electromagnetic based on the subsection control approach," Mathematical Problems in Engineering, vol. 2021, Article ID 6660393, 9 pages, 2021

[21] H. Zhou, Y. Yao, and C. Liu, "Feasibility of signal enhancement with multiple grounded-wire sources for a frequency-domain electromagnetic survey," Geophysical Prospecting, vol. 66, no. 4, pp. 818-832, 2018. 